\title{
Cultural method for large-scale screening for Chlamydia trachomatis genital infection
}

\author{
H MALLINSON, S SIKOTRA, OP ARYA* \\ From the Regional Public Health Laboratory, Fazakerley Hospital, Liverpool L9 $7 A L$ and *University \\ Department of Venereology, Royal Liverpool Hospital, Liverpool L7 8 XP
}

SUMMARY Idoxuridine-treated McCoy cells grown as monolayers in 96 well microplates provide a convenient method for the isolation of Chlamydia trachomatis. Staining of infected monolayers with $\omega_{\perp}^{\dot{\omega}}$ periodic acid-Schiff reagent (PAS) allows easy recognition of $C$ trachomatis inclusions without the - v need for dark-ground microscopy. By this method 384 clinical specimens can be examined con $-\frac{V}{N}$ currently. It is sufficiently sensitive to form the basis of a chlamydial culture service for patientso attending Sexually Transmitted Diseases (STD) Clinics.

Chlamydia trachomatis causes neonatal conjunctivitis, has become accepted as a cause of "nonspecific" urethritis and post-gonococcal urethritis in men as well as "non-specific" genital infection in women, and has been increasingly associated with other diseases of the genital and respiratory tracts. ${ }^{1}$ Recently Willcox et al., ${ }^{2}$ pointing out the large reservoir of untreated chlamydial infection in female patients attending venereal diseases clinics, have reviewed the need for a chlamydial culture service. They conclude that facilities for the isolation of $C$ trachomatis should be an essential aid in the management of women attending Sexually Transmitted Diseases (STD) Clinics and that male patients would also benefit if such facilities were readily available.

The current methods for the isolation of $C$ trachomatis most commonly involve inoculation of McCoy cell monolayers grown on individual glass coverslips, centrifugation, incubation and detection of intracytoplasmic chlamydial inclusions when the Giemsa-stained monolayers are examined by darkground microscopy. Unfortunately the need to centrifuge and to stain individual cultures and the need to view several hundred microscopic fields per coverslip make the method both time-consuming and labour-intensive. ${ }^{3}$ This complexity of the isolation method may account in part for the observation by Willcox et al. ${ }^{2}$ that "those few clinics with laboratory facilities for the isolation of $C$ trachomatis tend to be looked on as research centres and are not generally regarded as providing an essential aid to the diagnosis and management of the most common sexually transmitted disease."

Accepted for publication 19 November 1980
We describe a technique for the isolation of $\frac{\bar{C}}{C^{\prime}}$ trachomatis with idoxuridine-treated McCoy cells $s_{\infty}$ grown in 96 well microplates, stained after incubation ${ }^{-}$ with periodic acid-Schiff reagent (PAS) which allows the examination of large numbers of clinical speci-s mens quickly and conveniently without the need for dark-ground microscopy. The sensitivity of the new technique has been compared with that of $\frac{2}{2}$ method based on Giemsa staining of McCoy cell coverslip cultures which has given good isolationg rates of $C$ trachomatis over a five-year period.

\section{Material and methods}

CLINICAL SPECIMENS FOR C TRACHOMATIS ISOLATION

Endocervical swabs from women and endourethraB swabs from male patients were collected at the STD Clinic, St James' Hospital, Birkenhead.

Stock culture of C trachomatis for laboratory studie This isolate from the cervix, designated 4394CP3, was used at the third passage in idoxuridine-treateos McCoy cells.

\section{Chlamydia transport medium (TM)}

199 medium (Wellcome TC22) with the addition of $10 \%$ sorbitol (see results section); $0.5 \%$ glucose ${ }^{+}$ $10 \%$ fetal calf serum; $100 \mu \mathrm{g} / \mathrm{ml}$ each of vancomycin streptomycin and mycostatin; and $0.11 \%$ sodiun bicarbonate, distributed in $2.5 \mathrm{ml}$ amounts ir screw-capped bijoux bottles. Transport medium wa\$ orange-yellow in colour (discarded if pink) and was held at $+4^{\circ} \mathrm{C}$ before use. Swabs in TM weres 
recooled at $+4^{\circ} \mathrm{C}$ then carried to the laboratory in a vacuum flask.

\section{McCoy cell culture media}

The media used were based on Eagles MEM 10x concentrate (Gibco-Biocult, Cat No 143E) with pH adjusted by an initial addition of $0.4 \%$ of $5 \mathrm{~N}$ sodium hydroxide.

$M c$ Coy cell growth medium (GM): Eagles MEM with $1 \%$ glutamine $(200 \mathrm{mM}) ; 1 \%$ MEM vitamins (Gibco-Biocult $100 x$ concentrate); $100 \mu \mathrm{g} / \mathrm{ml}$ vancomycin; $100 \mu \mathrm{g} / \mathrm{ml}$ streptomycin; $10 \mu \mathrm{g} / \mathrm{ml}$ gentamicin, $10 \%$ fetal calf serum and $0.22 \%$ sodium bicarbonate.

McCoy cell maintenance medium (MM): Growth medium as above with additional $0.5 \%$ glucose and $2 \cdot 5 \mu \mathrm{g} / \mathrm{ml}$ amphotericin B.

\section{McCoy cell stock cultures}

McCoy cells were grown in $125 \mathrm{ml}$ medical flat bottles seeded at a rate of $6 \times 10^{4}$ cells $/ \mathrm{ml}$ with $20 \mathrm{ml}$ GM. They were used after three or four days incubation at $37^{\circ} \mathrm{C}$-that is, when just confluent.

\section{IDU treatment of McCoy cells}

To prepare coverslip and microplate cultures suitable for infection with $C$ trachomatis, McCoy cells were grown at $37^{\circ} \mathrm{C}$ for 3-5 days (usually 4) in the presence of IDU using methods adopted from those of Reeve et al. ${ }^{4}$

Idoxuridine (IDU) stock solution: 5-iodo-2-deoxyuridine (Sigma Chemicals) at $1.25 \mathrm{mg} / \mathrm{ml}$ in a mixture of dimethyl sulphoxide $10 \%$, deionised water $90 \%$ stored at $4^{\circ} \mathrm{C}$ in the dark and diluted $1 / 50$ in McCoy cell growth medium (GM) for working strength.

McCoy cell coverslip cultures: Prepared by adding $6 \times 10^{4}$ cells suspended in $1 \mathrm{ml}$ of GM supplemented with $25 \mu \mathrm{g} / \mathrm{ml}$ IDU to a Searle E10 plastic tube containing a Chance, thickness " 0 ," $12 \mathrm{~mm}$ diameter glass coverslip.

McCoy cell microplate cultures: Prepared by adding $7.5 \times 10^{3}$ cells suspended in $0.25 \mathrm{ml}$ of $\mathrm{GM}$ containing $25 \mu \mathrm{g} / \mathrm{ml}$ IDU to each of the 96 flat-bottomed wells of the plate (Flow Laboratories Cat No 76-202-05) which was then sealed with non-toxic, adhesive sealing film (Flow Laboratories Cat No 76-402-05).

GROWTH OF C TRACHOMATIS

McCoy cell coverslip and microplate cultures were first drained to remove GM containing IDU and were then treated as follows:

Coverslip cultures in tubes were refed with 1.5 $\mathrm{ml}$ MM followed by either $0.5 \mathrm{ml}$ of TM where clinical specimens were to be inoculated or $0.5 \mathrm{ml}$ of MM containing $C$ trachomatis (isolate 4394CP3) for laboratory studies. Inoculated tubes were centrifuged at $34^{\circ} \mathrm{C}$ at $1900 \mathrm{~g}$ for one hour and incubated at $37^{\circ} \mathrm{C}$ for $72 \mathrm{~h}$.

Microplate cultures were refed with $0.2 \mathrm{ml} \mathrm{MM}$ per well followed by either $0.1 \mathrm{ml}$ TM for isolation from clinical specimens or $\mathbf{0 . 1 \mathrm { ml }} \mathrm{MM}$ containing $C$ trachomatis (4394CP3) for other tests. Inoculated plates were resealed and then spun in microplate carriers (similar to those available from Dynatech Laboratories Ltd Cat No MI8E) at $2200 \mathrm{rpm} 1450 \mathrm{~g}$ for three hours in an MSE Mistral 4L centrifuge at $34^{\circ} \mathrm{C}$. Plates were incubated at $37^{\circ} \mathrm{C}$ for $72 \mathrm{~h}$.

\section{STAINING OF CHLAMYDIAL INCLUSIONS}

\section{Giemsa stain}

Coverslip cultures were stained $72 \mathrm{~h}$ after inoculation by the Giemsa method of Johnson. ${ }^{5}$ Stained coverslips were dislodged by piercing the plastic tube with a hot wire, dehydrated in acetone (5-10 s) cleared in xylol and mounted cell sheet downwards in XAM mountant on thin dark-ground microscope slides. They were observed by dark-ground microscopy using $\times 200$ or $\times 400$ magnification.

\section{Periodic acid-Schiff (PAS) stain}

Microplate cultures were stained in baths in which the whole plate could be immersed.

Plates were: fixed in methanol for $5 \mathrm{~min}$ (to avoid the release of infectious material, sealing film was removed with the plate already immersed in the methanol bath); washed and allowed to stand in tap water for $30 \mathrm{~s}$; "oxidised" in $1 \%$ aqueous periodic acid (BDH) for $10 \mathrm{~min}$; washed for $5 \mathrm{~min}$ using 3 changes of distilled water; stained in Schiff reagent (Hopkin \& Williams) for $20 \mathrm{~min}$; washed repeatedly in tap water ( 5 changes in $5 \mathrm{~min}$ ) stained with Harris haematoxylin (Paramount Reagents) for $\frac{1}{2}-1 \mathrm{~min}$; washed in warm tap water for $1 \mathrm{~min}$; shaken dry. Each well then received 2-3 drops of Aquamount (Hopkin \& Williams).

Coverslip cultures were stained by the same procedure but were dehydrated and mounted on microscope slides as described for Giemsa-stained coverslips. When coverslips and microplates were examined by transmitted illumination on an inverted microscope $(\times 100-\times 400$ magnification) tissue culture cells stained blue and chlamydial inclusions stained bright magenta. 
Results

PAS STAINING OF CHLAMYDIAL INCLUSIONS Periodic acid-Schiff (PAS) staining is a commonly used histological technique for the demonstration of glycogen. Application of the PAS stain to $C$ trachomatis infected McCoy cell monolayers revealed magenta intra-cytoplasmic inclusions showing features of the morphology of inclusions stained by the more usual Giemsa or iodine staining techniques (Figs 1, 2, and 3). Some inclusions (presumably rich in glycogen) showed little or no internal structure and stained a uniform magenta colour. In other cases inclusions had an overall granular appearance due to staining of individual elementary bodies. If required PAS-stained monolayers can be examined by dark-ground microscopy. Under these conditions background cells in the monolayer appeared pinkpurple, inclusions with a high glycogen content were bright red and more granular inclusions resembled the yellow fluorescent inclusions seen after Giemsa staining but with a superimposed red hue.

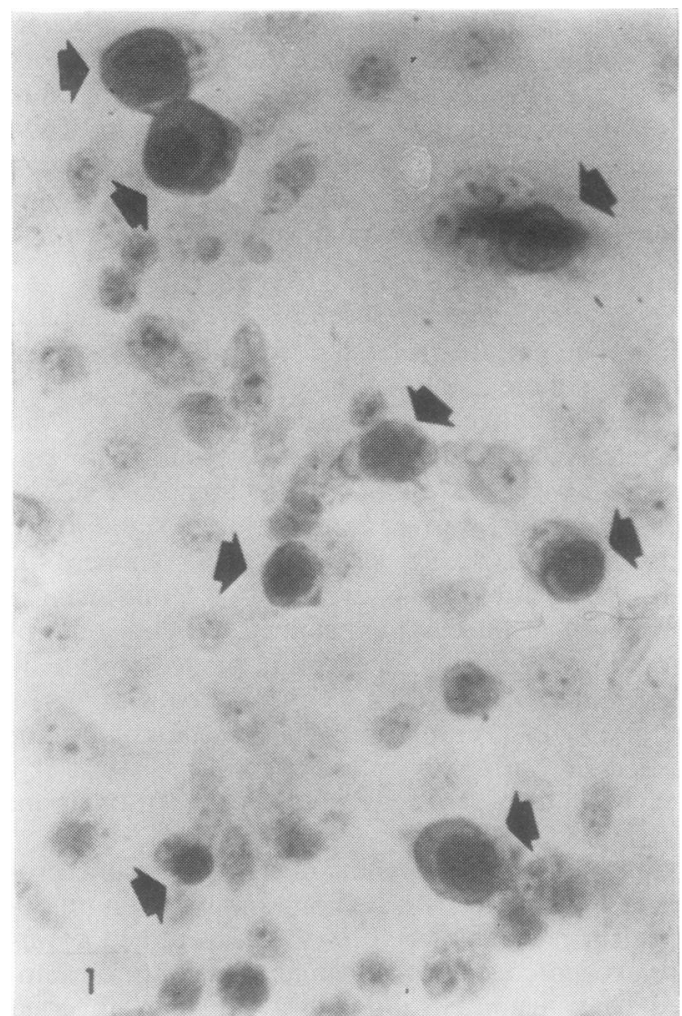

Fig. 1 Chlamydia trachomatis inclusions (arrowed) in McCoy cells. Periodic acid-Schiff stain $\times 200$ (original magnification).
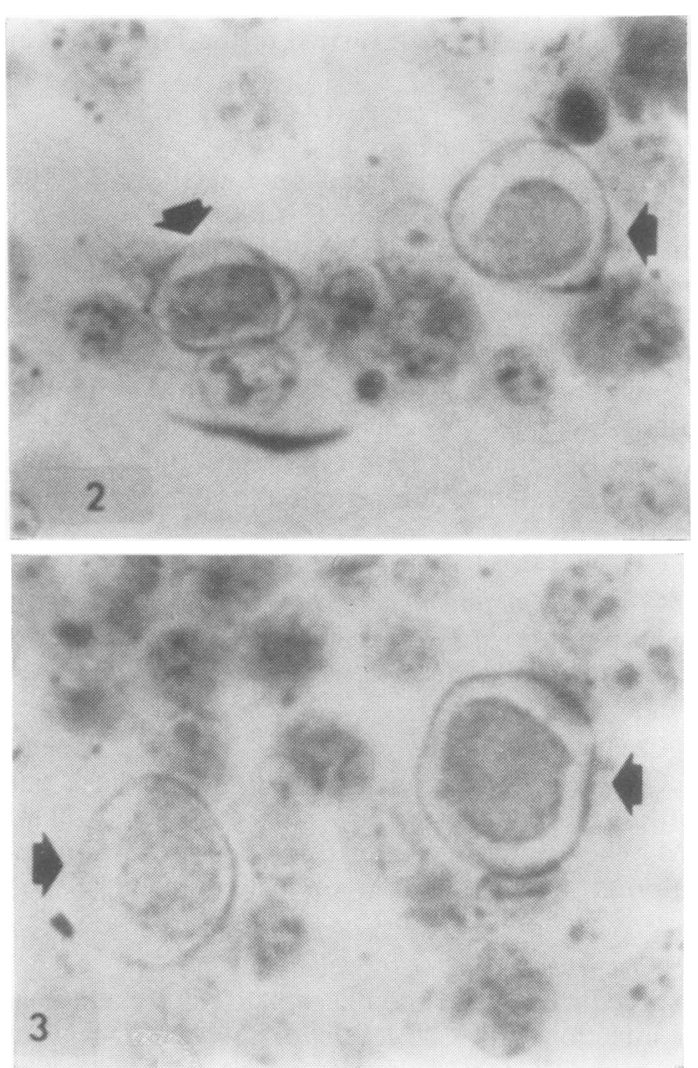

Figs 2 and 3 Chlamydia trachomatis inclusions (arrowed) in McCoy cells. Periodic acid-Schiff stain $\times 400$ (original magnification).

Table 1 shows results of three experiments com- $\frac{0}{7}$ paring counts on coverslips stained either with PAS. (examined using transmitted illumination) or with Giemsa (examined by dark-ground microscopy). Inclusion counts by the two staining methods were윽 comparable and altered little during the period 48 to $>$ $96 \mathrm{~h}$ after inoculation. When screening coverslips for evidence of infection, inclusions stained by PAS N were more readily apparent, being easily detected evenat a magnification as low $(\times 47)$ as that used to scan 0 tissue-cultures for viral cytopathic effects.

COMPARISON OF CHLAMYDIA INCLUSION COUNTS ON COVERSLIP AND IN

MICROPLATE WELLS

On seven separate occasions various dilutions of 0 $C$ trachomatis strain $4394 \mathrm{CP} 3$ were inoculated in parallel on to IDU-treated McCoy cells grown on $\stackrel{\odot}{\Phi}$ coverslips and in microplate wells.

Table 2 details the inclusion counts found aftero $72 \mathrm{~h}$ incubation when coverslips were stained with 
Table 1 Comparison of chlamydial inclusion counts following Giemsa or PAS staining of coverslips inoculated with C trachomatis strain $4394 C P 3$

\begin{tabular}{|c|c|c|c|c|c|}
\hline \multirow[t]{2}{*}{ Incubation time $(h)$} & \multicolumn{2}{|l|}{ Giemsa stain } & \multicolumn{2}{|l|}{$P A S$ stain } & \multirow{2}{*}{$\begin{array}{l}\text { Ratio of counts } \\
\text { Giemsa:PAS }\end{array}$} \\
\hline & Inclusion counts per coverslip & Average count & Inclusion counts per coverslip & Average count & \\
\hline $\begin{array}{r}48 \\
72 \\
96 \\
120 \\
\end{array}$ & $\begin{array}{c}38 / 57 / 35 \\
-/ 26 /- \\
51 / 54 / 49 \\
-/ 266 /- \\
\end{array}$ & $\begin{array}{l}42 \cdot 6 \\
26 \\
51 \cdot 3 \\
266 \\
\end{array}$ & $\begin{array}{c}57 / 64 / 48 \\
56 / 52 \\
73 /-/ 65 \\
213 / 484 / 397 \\
\end{array}$ & $\begin{array}{c}56 \cdot 3 \\
54 \\
69 \\
365 \\
\end{array}$ & $\begin{array}{l}1: 1 \cdot 3 \\
1: 2 \cdot 1 \\
1: 1 \cdot 4 \\
1: 1 \cdot 4 \\
\end{array}$ \\
\hline 72 & $105 / 115 / 95$ & 105 & $102 / 61 / 87 / 81$ & $82 \cdot 8$ & $1: 0 \cdot 8$ \\
\hline 72 & $\begin{array}{l}381 / 316 / 320 \\
247 / 241\end{array}$ & 301 & $\begin{array}{l}419 / 421 / 315 \\
275 / 396\end{array}$ & 365 & $1: 1 \cdot 2$ \\
\hline
\end{tabular}

Table 2 Comparison of chlamydial inclusion counts on coverslips and in microplate wells

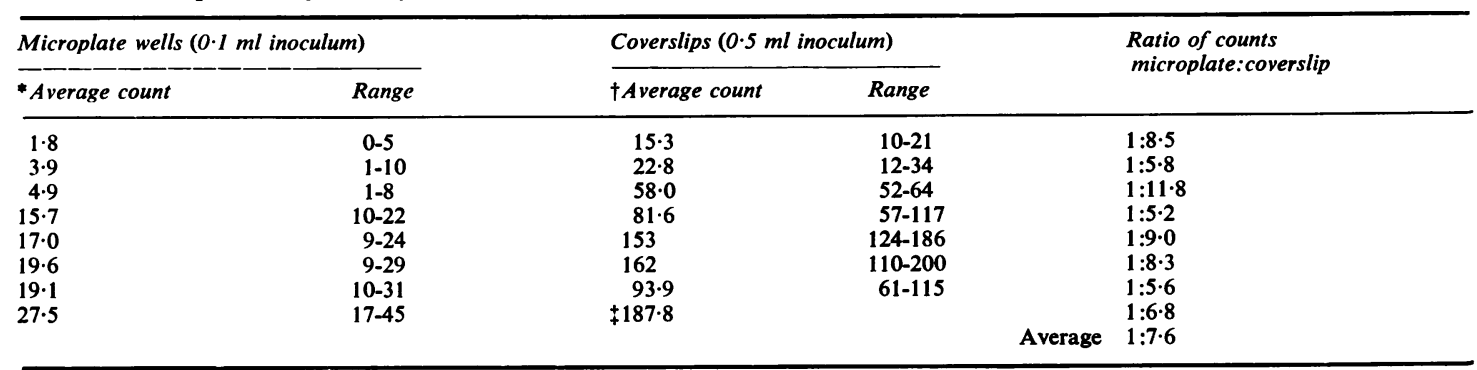

*Groups of 10 to 36 wells counted.

tGroups of 2 to 7 coverslips counted.

$\ddagger(93.9) \times 2$.

Giemsa and microplates were stained with PAS. With inocula of equivalent concentration, coverslip cultures receiving a $0.5 \mathrm{ml}$ inoculum showed on average 7.6 times (range $5 \cdot 2-11 \cdot 8$ ) higher inclusion counts than cultures in microplate wells which received a $0 \cdot 1 \mathrm{ml}$ inoculum.

\section{TRACHOMATIS ISOLATION RATES USING} COVERSLIP CULTURES: PROSPECTS FOR ISOLATION USING MICROPLATE CULTURES Over a period of three years, isolation of $C$ trachomatis was attempted from newly registered patients who had not received an antibiotic during the preceding month and who were first seen by one of us (OPA) at Monday or Thursday sessions of an STD clinic. Swabs sent to the laboratory in a transport medium containing $10 \%$ sorbitol were used to inoculate coverslip cultures and $C$ trachomatis was identified by Giemsa staining. With this technique $C$ trachomatis was isolated from the cervix of 158 $(33 \%)$ of 474 women and from the urethra of 191 $(46 \%)$ of 413 men with urethritis. In this patient population a quantitative survey of $C$ trachomatis in the genital tract (Mallinson et al. to be published) has shown that about half the chlamydia-positive swabs gave inclusion counts of more than 120 per coverslip. These would have been detected without difficulty in a microplate system with a sensitivity 7.6-fold lower than the coverslip technique. But it was clearly of interest to determine the proportion of clinical specimens likely to yield very low inclusion counts-that is, less than eight inclusions per coverslip - that would fail detection in a microplate system using an inoculum as small as $\mathbf{0 . 1} \mathrm{ml}$.

Reappraisal of stored coverslips showed that only four $(8.2 \%)$ swabs from 49 chlamydia-positive women and only five $(6.9 \%)$ swabs from 72 chlamydia-positive men gave counts of less than eight inclusions per coverslip (actual counts $2,3,3,4,4,6$, $6,7,7)$.

\section{RISK OF CROSS-CONTAMINATION}

Throughout the development of the microplate technique, uninoculated "control" wells adjacent to wells inoculated with $C$ trachomatis were examined for evidence of cross-contamination. $C$ trachomatis inclusions have not been seen in the uninoculated wells. When alternate wells of two microplates were inoculated with $C$ trachomatis (strain 4394CP3, titre $1.2 \times 10^{3}$ inclusion forming units $/ \mathrm{ml}$ on coverslips) no evidence of cross-contamination was found in the uninoculated wells of the plates. 
CULTURE OF C TRACHOMATIS FROM

CLINICAL SPECIMENS IN MICROPLATES

Effect of sorbitol in transport medium Table 3 shows that when sorbitol was present during culture of $C$ trachomatis (strain 4394CP3) in McCoy cells, there was a reduction in inclusion count. A similar effect of sorbitol on inclusion counts was also noted when clinical specimens containing $C$ trachomatis were tested. Specimens for $C$ trachomatis isolation are routinely sent to our laboratory in a transport medium containing $10 \%$ sorbitol as recommended by Richmond. ${ }^{6}$ With this transport medium, the procedures described here for inoculating clinical specimens would result in final sorbitol concentrations in the culture medium of $2.5 \%$ in coverslip cultures and $3.3 \%$ in microplate wells. It can be seen from the data of Table 3 that this higher concentration of sorbitol $(3.3 \%)$ in microplate wells is particularly detrimental to inclusion counts. In our experience sorbitol shows a protective effect when specimens are to be stored frozen but is otherwise of no benefit in the transport of clinical material. We therefore suggest that transport medium without sorbitol be used for specimens to be examined by microplate culture.

Effect of clinical specimens A small series of clinical specimens collected into transport medium without sorbitol has been inoculated into microplate cultures and after incubation stained by PAS. Some specimens contained particulate materials which adhered to the monolayer and which stained magenta with PAS. $C$ trachomatis inclusions were, however, easily distinguished from this "non-specific" material by their characteristic morphology and intracytoplasmic location. Of 20 cervical swabs collected in transport medium without sorbitol and examined by inoculation of one coverslip and four microplate wells, 14 swabs were negative and six swabs were shown to contain $C$ trachomatis by both methods of culture. The least positive specimen which gave only eight inclusions on the whole coverslip produced a single inclusion in $\frac{0}{\overrightarrow{5}}$ two of the four inoculated microplate wells.

\section{Discussion}

Although it is a common histological technique, $\frac{\bar{C}}{\bar{D}}$ periodic acid-Schiff (PAS) staining has not pre- $\widehat{\Phi}$ viously, so far as we are aware, been described as a means of demonstrating $C$ trachomatis inclusions in ${ }^{\infty}$ tissue culture monolayers. PAS-stained inclusions $\vec{O}$ are readily seen by bright field microscopy whereas $\vec{\exists}$ Giemsa-stained inclusions are recognisable with ease ${ }_{\sigma}^{\omega}$ only by dark-ground microscopy with the attendant requirement of placing oil between the microscope slide and the dark-ground condenser. PAS may be + preferred to iodine-staining since, as well as staining. glycogen-rich inclusions intensely, it appears to show $\vec{N}$ up the elementary bodies in inclusions containingo little glycogen. At $72 \mathrm{~h}$ incubation, PAS inclusion? counts are similar to those found by Giemsa- $c$ staining. In screening patients for $C$ trachomatis infection we have routinely stained infected mono- $\vec{\theta}$ layers with Giemsa after $72 \mathrm{~h}$ incubation and it wouldo appear that a change to PAS-staining would not reduce the sensitivity of the isolation system.O Monolayers stained by PAS can be mounteds permanently when coverslips are used, or semipermanently in microplate wells, and then read ato leisure. Amorphous materials which stain with PASQ may be present occasionally in cultures inoculated $\overrightarrow{0}$ with clinical specimens but these do not present a problem as $C$ trachomatis inclusions are readily? distinguished by their characteristic morphology and position in the cytoplasm of infected McCoy cells The PAS stain therefore offers several advantages over Giemsa and iodine-staining and may prove more convenient for those laboratories which regularlys examine coverslips for $C$ trachomatis inclusions.

Perhaps of equal importance, PAS staining provides a means of detecting $C$ trachomatis in? clusions present in monolayers grown in microplates wells which are inaccessible to dark-ground micro

Table 3 Influence of sorbitol on inclusion count of C trachomatis grown in McCoy cells

\begin{tabular}{|c|c|c|c|c|}
\hline \multirow[t]{3}{*}{ Final percentage sorbitol in culture medium } & \multicolumn{4}{|c|}{ Average inclusion counts $(\%)$} \\
\hline & \multicolumn{2}{|c|}{ Microplate cultures } & \multicolumn{2}{|c|}{ Coverslip cultures } \\
\hline & Expt 1 & Expt 2 & Expt 3 & Expt 4 \\
\hline \multirow{7}{*}{$\begin{array}{l}0 \\
0 \cdot 6 \\
0.8 \\
1 \cdot 3 \\
1 \cdot 7 \\
1 \cdot 9 \\
2 \cdot 2 \\
2 \cdot 5 \\
3 \cdot 3\end{array}$} & $22 \cdot 7(100)$ & $19 \cdot 6(100)$ & $162(100)$ & $58(100)$ \\
\hline & & $17 \cdot 7(90)$ & $153(94)$ & \\
\hline & & 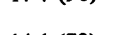 & $124(77)$ & \\
\hline & $18 \cdot 5(82)$ & $14 \cdot 1(72)$ & & \\
\hline & & & $120(74)$ & \\
\hline & $15 \cdot 5(68)$ & $8 \cdot 2(42)$ & $92(57)$ & $28 \cdot 5$ (49) \\
\hline & $5 \cdot 8(26)$ & $4.5(23)$ & & \\
\hline
\end{tabular}


scopy. Culture of $C$ trachomatis by a "micro cell culture method" has been reported previously" but this group (Boston, USA) have used microplates simply as culture vessels for monolayers grown on small glass coverslips which after inoculation and incubation were removed for staining by immunofluorescence ${ }^{8} 9$ or Giemsa. ${ }^{10}$ The alternative technique, presented here, of using monolayers attached directly to the flat base of microplate wells has the advantage that the 96 separate wells of the plate can be treated as a single unit during staining and microscopy.

Cross-contamination occurring between the closely-spaced wells of a microplate could lead to "false-positive" $C$ trachomatis isolation results. This danger would be accentuated if subculture of hightitre fluids from well to well were a necessary part of the culture procedure. However, our method carries a risk of cross-contamination only at the stage of initial inoculation of the wells and in practice this has been avoided by use of careful technique.

With available equipment the microplate culture method and PAS staining could be applied to the examination of 384 (4 plates $\times 96$ wells) clinical specimens concurrently. Estimates of the potential sensitivity of this technique for detecting chlamydiapositive patients attending STD clinics have been based on comparison with a coverslip culture which has given good isolation rates of $C$ trachomatis over several years of routine use. In a stringent laboratory assessment, in which a $0.1 \mathrm{ml}$ inoculum in microplate wells was compared with a $0.5 \mathrm{ml}$ inoculum on coverslip cultures, inclusion counts in the coverslip method exceeded those in the microplate method by a factor of $7 \cdot 6$. This reduced sensitivity of the microplate method was also apparent when a small series of clinical specimens was examined.

The higher counts on coverslips are largely explained by the greater volume of inoculum used: $0.5 \mathrm{ml}$ as compared to $0.1 \mathrm{ml}$ in microplate wells. The remaining 2.6-fold lower sensitivity of the microplate method must be attributed to the altered conditions of culture and was possibly due to the lower centrifugal force $(1450 \mathrm{~g}$ instead of $1900 \mathrm{~g}$ ) after inoculation of microplate cultures.

It may be expected that clinical specimens showing between one and seven inclusions in the coverslip method will probably give false-negative results by the microplate method. We have found, however, that amongst chlamydia-positive STD patients the proportion giving such low inclusion counts is small $(8.2 \%$ women, $6.9 \%$ men) and these estimates are supported by the work of others. ${ }^{11}$ Thus, if applied to women attending STD clinics, the microplate method should detect $91.8 \%$ of those in whom $C$ trachomatis infection can be demonstrated by conventional methods and possibly an even higher proportion if more than one well per specimen were inoculated.

The call by Willcox et $a .^{2}$ for a chlamydial culture service for women attending STD clinics has been supported recently by Arya et al. ${ }^{12}$ and Richmond et al. ${ }^{13}$ The alternative approach of serological detection of $C$ trachomatis infections was reviewed by Taylor-Robinson and Thomas ${ }^{1}$ who found that apart from a few circumstances this did not help in diagnosis. These same workers state that a culture service would be of value in selected STD patients provided it withstood examination in terms of "cost-effectiveness" or "cost-usefulness." We feel that the microplate-PAS technique offers the balance of sensitivity and efficiency needed to provide such a chlamydial culture service.

We thank Mr AF Lloyd for his advice on staining methods and for his help, together with Mr CI Ashton, with photography. We are grateful to $\mathrm{Mr} \mathbf{J}$ Peel for producing the carriers used to centrifuge microplates.

\section{References}

${ }^{1}$ Taylor-Robinson D, Thomas BJ. The role of Chlamydia trachomatis in genital tract and associated diseases. $J$ Clin Pathol 1980;33:205-33.

${ }^{2}$ Willcox JR, Fisk PG, Barrow J, Barlow D. The need for a chlamydial culture service. Br J Vener Dis 1979;55:281-3.

${ }^{3}$ Hobson D. Tissue culture procedures for the isolation of Chlamydia trachomatis from patients with non-gonococcal urethritis. In: Hobson D, Holmes KK, eds. Non-gonococcal urethritis and related infections. Washington DC: American Society for Microbiology, 1977: 286-94.

${ }^{4}$ Reeve P, Owen J, Oriel JD. Laboratory procedures for the isolation of Chlamydia trachomatis from the human genital tract. J Clin Pathol 1975;28:910-4.

5 Johnson FWA. A comparison of staining techniques for demonstrating group A chlamydia in tissue culture. Medical Laboratory Technology 1975;32:233-8.

${ }^{6}$ Richmond SJ. The isolation of Chlamydia subgroup A (Chlamydia trachomatis) in irradiated McCoy cells. Medical Laboratory Technology 1974;31:7-9.

${ }^{7}$ McComb DE, Puzniak CI. Micro cell culture method for isolation of Chlamydia trachomatis. Appl Microbiol 1974; 28:727-9.

8 McCormack WM, Alpert S, McComb DE, Nichols RL, Sermine DZ, Zinner SH. Fifteen month follow-up study of women infected with Chlamydia trachomatis. $N$ Engl $J$ Med 1979;300:123-5.

${ }^{9}$ Hammerschlag MR, Anderka M, Semine DZ, McComb D, McCormack WM. Prospective study of maternal and infantile infection with Chlamydia trachomatis. Pediatrics $1979 ; 64: 142-8$.

10 Rota TR. Techniques for culturing and determining antimicrobial susceptibility of Chlamydia trachomatis. Arch Androl 1980;4:63-9.

11 Johnson FWA, Hobson D. Factors affecting the sensitivity of replicating McCoy cells in the isolation and growth of Chlamydia A (TRIC agents). J Hyg (Camb) 1976;76: 441-51. 
12 Arya OP, Mallinson H, Goddard AD. Epidemiological and clinical correlates of chlamydial infection of the cervix. Br J Vener Dis 1981 ;57:118-24.

${ }^{13}$ Richmond SJ, Paul ID, Taylor PK. Value and feasibility of screening women attending STD clinics for cervical chlamydial infections. Brit J Vener Dis 1980;56:92-5.

T

Requests for reprints to: $\mathrm{Dr} H$ Mallinson, Regiona $\vec{P}$ Public Health Laboratory, Fazakerley Hospital, Liver $\vec{F}$ pool L9 7AL, England. 